\title{
Sheep can be used as animal model of regional myocardial remodeling and controllable work
}

\author{
Jürgen Duchenne ${ }^{1}$, Piet Claus ${ }^{1}$, Efstathios D. Pagourelias ${ }^{1}$, Razvan O. Mada, \\ Joeri Van Puyvelde ${ }^{2}$, Kathleen Vunckx ${ }^{3}$, Eric Verbeken ${ }^{4}$, Olivier Gheysens ${ }^{3}$, \\ Filip Rega ${ }^{2}$ Jens-Uwe Voigt ${ }^{1}$
}

\begin{abstract}
${ }^{1}$ Department of Cardiovascular Sciences and Department of Cardiovascular Diseases, KU Leuven - University of Leuven and University Hospitals Leuven, Leuven, Belgium

${ }^{2}$ Department of Cardiovascular Sciences and Department of Cardiothoracic Surgery, KU Leuven - University of Leuven and University Hospitals Leuven, Leuven, Belgium

${ }^{3}$ Department of Imaging and Pathology and Department of Nuclear Medicine, KU Leuven - University of Leuven and University Hospitals Leuven, Leuven, Belgium

${ }^{4}$ Department of Imaging and Pathology and Department of Pathology, KU Leuven

- University of Leuven and University Hospitals Leuven, Leuven, Belgium
\end{abstract}

\begin{abstract}
Background: Pacing the right heart has been shown to induce reversible conduction delay and subsequent asymmetric remodeling of the left ventricle (LV) in dogs and pigs. Both species have disadvantages in animal experiments. Therefore the aim of this study was to develop a more feasible and easy-to-use animal model in sheep.

Methods: Dual-chamber (DDD) pacemakers with epicardial leads on the right atrium and right ventricular free wall were implanted in 13 sheep. All animals underwent 8 weeks of chronic rapid pacing at $180 \mathrm{bpm}$. Reported observations were made at $110 \mathrm{bpm}$.

Results: DDD pacing acutely induced a left bundle branch block (LBBB) - like pattern with almost doubling in QRS width and the appearance of a septal flash, indicating mechanical dyssynchrony. Atrial pacing (AAI) resulted in normal ventricular conduction and function. During 8 weeks of rapid DDD pacing, animals developed LV remodeling (confirmed with histology) with septal wall thinning (-30\%, $p<0.05)$, lateral wall thickening $(+22 \%, p<0.05)$, LV volume increase $(+32 \%, p<0.05)$, decrease of $L V$ ejection fraction $(-31 \%, p<0.05)$, and functional mitral regurgitation. After 8 weeks, segmental pressurestrain-loops, representing regional myocardial work, were recorded. Switching from AAI to DDD pacing decreased immediately work in the septum and increased it in the lateral wall (-69 and $+41 \%$, respectively, $p<0.05)$. Global LV stroke work and $d P / d t m a x$ decreased (-27\% and $-25 \%$, respectively, $p<0.05)$.

Conclusions: This study presents the development a new sheep model with an asymmetrically remodeled LV. Simple pacemaker programing allows direct modulation of regional myocardial function and work. This animal model provides a new and valuable alternative for canine or porcine models and has the potential to become instrumental for investigating regional function and loading conditions on regional LV remodeling. (Cardiol J 2019; 26, 4: 375-384)
\end{abstract}

Key words: animal model, regional myocardial work, pacing, dyssynchrony, remodeling

Address for correspondence: Prof. Dr. Jens-Uwe Voigt, Department of Cardiovascular Diseases, University Hospitals Leuven, Herestraat 49, 3000 Leuven, Belgium, tel: +32 16 349016, fax: +32 16 344240, e-mail: jens-uwe.voigt@uzleuven.be 


\section{Introduction}

Left ventricular (LV) remodeling, as observed in pathologies with inhomogeneous myocardial function such as conduction delays, is still not fully understood, and animal models with conduction delays may facilitate further research in this field. Animal models where regional function can be modulated in a reversible way are of particular interest for studying the effect of regional function and work on regional remodeling.

Traditionally, large animal models, such as dogs, pigs and sheep are used in translational cardiovascular research, because of their similarity to human anatomy and physiology [1]. Dogs are considered the most suitable species [2] as their conduction system as well as the consecutive remodeling of the myocardium is very similar to humans. On the other hand, dog models are expensive and present ethical problems in many countries [3, 4]. Pig models are cheaper and easier to work with but grow very rapidly which makes them less suited for remodeling studies with follow up examinations [3, 4]. Furthermore, they are highly susceptible to ventricular arrhythmias generating a costly risk for long term experiments [5]. Minipigs might be an alternative [6], but costs, availability and their smaller hearts will prevent their use in many cases. Sheep would be an elegant alternative for remodeling experiments as they are cheap, have a slow growth rate and show a docile behaviour. However, intraventricular conduction in sheep is fast, so some authors have questioned their suitability for studies involving modulation of the conduction system [2]. However, so far, little is known about the usability of sheep in this field.

Conduction delays such as left bundle branch block (LBBB) have been shown to lead to a typical asymmetrical pattern of remodeling: thickening of the postero-lateral LV wall and thinning of the septum [7, 8]. Previous animal studies specifically aiming at asymmetric remodeling and work distribution have applied ablation to create a LBBB, thereby accepting the irreversible damage of the conduction system, and requiring up to 6 months to develop significant remodeling [9]. Other studies applied 3-6 months of LV pacing at physiological heart rate, however thereby not resembling the wall thickness distribution normally seen in LBBB $[10,11]$. Pacing the right heart with leads on/in the right atrium (RA) and right ventricle (RV) should provide the desired remodeling, with thinned septum and thickened lateral wall. Additionally, the pacing effect is reversible, as it allows switching between regular LV conduction (RA pacing only, or AAI mode) and LBBB-like conduction and myocardial function pattern (sequential RA and RV pacing, or DDD mode). One can thereby directly compare myocardial mechanics and hemodynamics in and between both settings. By applying pacing rapidly, remodeling effects have been shown to be possible within a few weeks [12-15].

To our knowledge, no studies have created a sheep model that allows for reversible conduction delay and controllable regional myocardial work in a remodeled LV. Sheep would allow for both an easy-to-use, slow growing and relatively cheap large animal model in order to facilitate future studies on the pathophysiology of myocardial remodeling and its effect on the regional myocardial function. Therefore, the aim of the present study was to investigate whether such an model could be created.

\section{Methods}

\section{Animal model outline}

In this study, 13 juvenile female SwifterCharolais sheep (average body weight $38 \pm 3 \mathrm{~kg}$; average age: 12 months) were implanted with a dual-chamber pacemaker. Ewes were used because of their more docile behaviour compared to rams. All animals were allowed to recover from the implantation procedures for 3 days. After recovery, baseline magnetic resonance imaging (MRI) and echocardiography were performed on the same day. Immediately after baseline imaging, rapid DDD pacing was started to induce $L V$ remodeling with an LBBB-like morphology and function. Weekly echocardiograms were performed to monitor the remodeling process. After 8 weeks of rapid pacing, MRI and echocardiography were repeated. Finally, invasive hemodynamic measurements in the catheterization lab, as well as simultaneous echocardiography were performed to characterize stroke work and regional myocardial work. Pilot experiments were used to optimize the experimental protocol (Suppl. material). The timeline of the protocol can be found in Figure 1.

All procedures performed in this study involving animals were in accordance with the ethical standards of the institution (project number P146/2012) and complied with the European Commission Directive 2010/63/EU. Animals were supplied by the KU Leuven Zoötechnisch Centrum. 


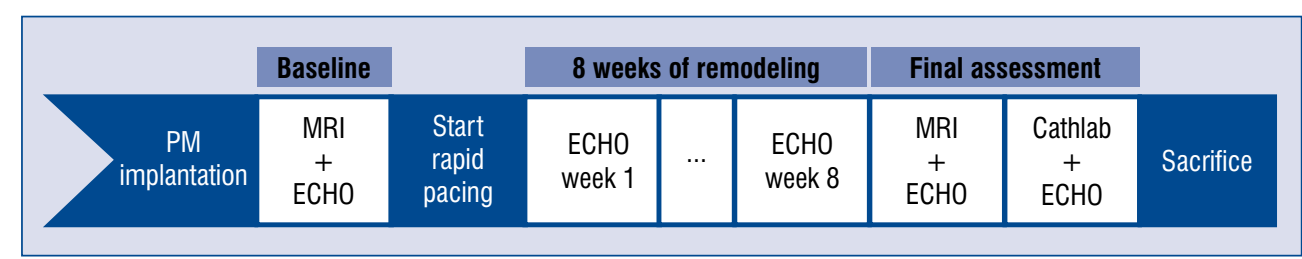

Figure 1. Timeline of the experimental protocol. Rapid pacing was at $180 \mathrm{bpm}$ and all imaging procedures and hemodynamic measurements were done at 110 bpm; ECHO - echocardiography; MRI — magnetic resonance imaging; $\mathrm{PM}$ - pacemaker.

\section{Pacemaker implantation}

In brief, animals were implanted with a dual-chamber pacemaker (Adapta L DDDR; Medtronic, Heerlen, The Netherlands) and epicardial pacemaker leads (Capsure EPI; Medtronic) on the RV free wall and RA, under general isoflurane anaesthesia. After implantation of the RV lead, we searched for signs of pacing induced LBBB-like activation pattern. Such patterns include QRS-width increase on the surface electrocardiogram (ECG), as well as pre-ejection septal flash in the intraoperative transthoracic echocardiogram (Suppl. Fig. 1, arrows). A detailed description of the pacemaker implantation procedure can be found in Supplemental material.

Imaging procedures at baseline setting Animal preparation for imaging

For all imaging procedures, animals were anesthetized using the same protocol as during the implantation procedure. The pacemaker was set to a fixed and reproducible heart rate of $110 \mathrm{bpm}$ (approximately $10 \mathrm{bpm}$ above the intrinsic heart rate) and data were acquired in both AAI and DDD mode (randomly determined). Images were acquired under apnoea by discontinuing ventilation, followed by a recovery period.

\section{Cardiac magnetic resonance imaging}

After the recovery period following pacemaker implantation, and before starting rapid pacing, a baseline cardiac MRI was performed. MRI acquisitions were performed to assess LV morphology and function, left atrium (LA) volume, and mitral valve regurgitation. Full methodological details on the MRI examinations can be found in the Supplemental material.

\section{Echocardiography}

Baseline echocardiography was performed on the same day as the baseline MRI. Echocardiographic assessment was focused on LV dimen- sions: wall thicknesses and cavity diameters. Full methodological details on the echocardiographic examinations can be found in the Supplemental material.

\section{Pacing protocol}

Immediately after baseline imaging, sequential RA and RV pacing (DDD) was started at a rate of $180 \mathrm{bpm}$. The rapid DDD pacing was maintained for 8 weeks, and was only lowered to $110 \mathrm{bpm}$ for all imaging and hemodynamic procedures. The atrio-ventricular delay was programed to $30 \mathrm{~ms}$ to guarantee complete ventricular capture from the site of pacing. Proper pacemaker function, pacing thresholds, and sensitivity were checked each week and adjusted when necessary.

\section{Imaging procedures during remodeling process}

Echocardiography was repeated weekly over a period of 8 weeks in duration in order to monitor the LV remodelling process, following the same protocol as during the baseline setting.

\section{Imaging procedures during \\ final assessment}

After the remodelling period of 8 weeks, both cardiac MRI and echocardiography were repeated on the same day, using the same protocol as during the baseline setting.

\section{Cathlab measurements during final assessment Animal preparation for invasive hemodynamic assessment}

One day after the imaging procedures during final assessment, hemodynamic measurements were performed to assess acute changes between pacing modes. Full methodological details on the hemodynamic measurements can be found in the Supplemental material. 
Left ventricular pressure-volume loops: estimation of global myocardial function

Left ventricular pressure and volume signals were simultaneously acquired in both pacing modes: AAI and DDD. Pressure and volume signals were recorded from $30 \mathrm{~s}$ before until $30 \mathrm{~s}$ after switching between pacing modes. Data analysis was performed off-line with LabChart. LV pressure-volume loops, maximum rate of ventricular pressure development $\left(\mathrm{dP} / \mathrm{dt}_{\max }\right)$, mean arterial pressure (MAP), and systemic vascular resistance (SVR) were calculated from the recordings. Global stroke work was assessed from the pressurevolume loops, and calculated from the area of the respective loop using a custom made MATLAB script (MathWorks, Eindhoven, The Netherlands) (mloop, J.-U. Voigt, Leuven, Belgium).

\section{Left ventricular pressure-strain loops:} estimation of regional myocardial work

To assess regional myocardial work, echocardiography was performed simultaneously with invasive hemodynamic measurements in the catheterisation lab. Echocardiographic assessment was focused on mid-ventricular LV segmental circumferential deformation. Deformation was assessed using speckle tracking echocardiography, in order to analyze work in both pacing modes: AAI and DDD.

Segmental circumferential myocardial deformation curves were extracted from the echocardiography data and carefully time-aligned with simultaneous LV pressure recordings, using ECG signals from both recordings as a time reference. Regional myocardial work was assessed by the area of segmental pressure-strain loops [16-18].

On completion of the experiments, the animals were sacrificed under isoflurane anaesthesia with intravenous $160 \mathrm{mg} / \mathrm{kg}$ pentobarbital and $50 \mathrm{~mL}$ of saturated $\mathrm{KCl}$.

\section{Histological analysis}

Ten out of 13 animals had myocardial samples taken of both septal and lateral wall. Samples were sliced and stained for analysis of the number of transected cardiomyocytes per reference line, and the percentage content of cardiomyocytes, connective tissue and vessels for assessment of fibrosis. Full methodological details on the histologic examinations can be found in the Supplemental material.

\section{Statistical analysis}

Analysis was performed using SPSS Statistics 20 (IBM, Chicago, IL, USA). All continuous variables are expressed as mean \pm standard deviation (SD) if normally distributed, or otherwise by median \pm interquartile range. Normality was assessed using the Shapiro-Wilk test. All measurements were repeated three times, and averaged. To depict changes associated with remodeling, generalized linear models for repeated measurements with Greenhouse-Geisser correction were used, applying Bonferroni post-hoc analysis for defining within-measurement statistical significance. Paired sample t-tests were used to determine the effect of switching between pacing mode, and histology. Linear regression analysis was used to investigate correlation between remodeling parameters and mitral valve regurgitation score. Intra- and inter-observer variability analysis was assessed in all animals, using intra-class correlation coefficient (ICC), as was the correlation between wall thicknesses and diameters assessed by both MRI and echocardiography. Statistical significance was assumed at a two-tailed probability level of $\mathrm{p}<0.05$.

\section{Results}

Twelve out of 13 animals completed the full experimental period. One animal died before final hemodynamic measurements due to respiratory failure.

\section{Electromechanical effects of pacing Electrical effect}

No arrhythmias were induced during the pacing. AAI pacing did not induce any changes in QRS width compared to sinus rhythm ( $41 \pm 4$ vs. $42 \pm 4 \mathrm{~ms}$, $\mathrm{p}=\mathrm{NS})$. DDD pacing, however, caused an immediate $+83 \%$ increase in QRS duration compared to AAI (from $41 \pm 4$ to $75 \pm 4 \mathrm{~ms}$ at $110 \mathrm{bpm}$, $\mathrm{p}<0.0001)$. After 8 weeks of pacing, QRS width had further increased to $80 \pm 4 \mathrm{~ms}$ ( $+7 \%$ compared to baseline DDD pacing, $\mathrm{p}=0.021$ ), which is almost a doubling compared to baseline AAI pacing $(+95 \%$, $\mathrm{p}<0.0001)$.

\section{Mechanical effect}

M-mode echocardiography revealed a pronounced early septal bouncing pattern (septal flash) in all animals during the pre-ejection phase in DDD pacing (Fig. 2, arrows), indicative of LV mechanical dyssynchrony. No septal flash was observed in sinus rhythm or during AAI pacing.

Circumferential strain curves showed systolic shortening in both septal and lateral wall during sinus rhythm and in AAI mode. In contrast, during 


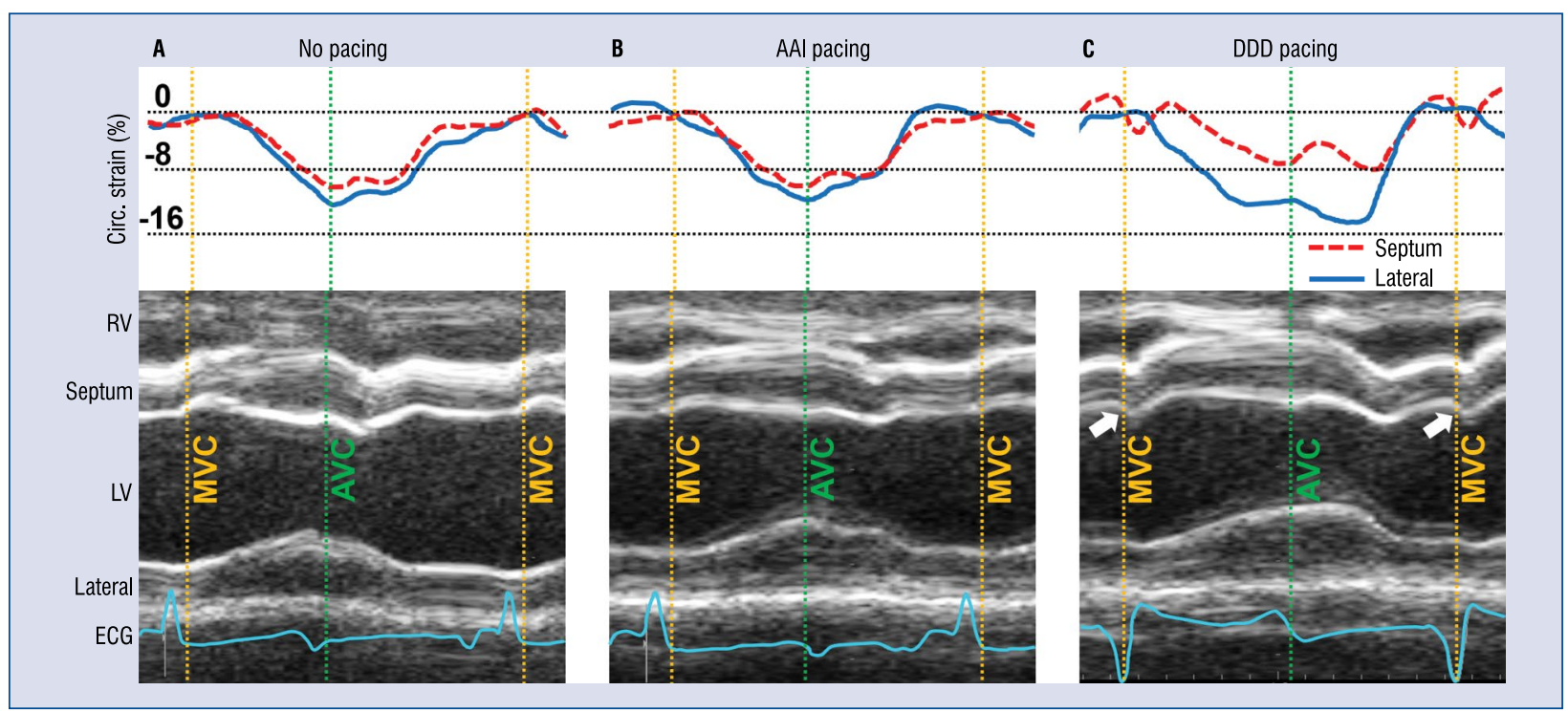

Figure 2. Representative septal and lateral circumferential strain traces (upper panel) and anatomical M-mode images (lower panel) of short-axis echocardiography images during pacemaker implantation; A. No pacing; B. During AAl pacing mode; C. During DDD pacing mode. Arrows indicates septal flash during DDD pacing. Vertical dashed lines indicate valve timings. Reported observations were made at 110 bpm; AVC - aortic valve closure; ECG - electrocardiogram; LV - left ventricle; MVC - mitral valve closure; RV — right ventricle.

DDD pacing, early shortening (septal flash) with subsequent systolic lengthening was seen in the septal region while the lateral wall shortened with delay during systole (Fig. 2).

\section{Left ventricular remodeling and functional deterioration with rapid pacing}

Within 8 weeks of rapid DDD pacing, echocardiography revealed that septal wall thickness decreased $-30 \%$ in end-diastole (ED) and $-31 \%$ in end-systole (ES) (from $9.2 \pm 1.0$ to $6.4 \pm 0.5 \mathrm{~mm}$, $\mathrm{p}<0.0001$ and from $10.3 \pm 0.9$ to $7.2 \pm 0.7 \mathrm{~mm}$, $\mathrm{p}<0.0001$, respectively) (Fig. 3A). At the same time, lateral wall thickness increased by $+22 \%$ in both ED and ES (from $9.5 \pm 0.6$ to $11.6 \pm 0.5 \mathrm{~mm}$, $\mathrm{p}=0.001$ and from $12 \pm 0.7$ to $14.6 \pm 0.5 \mathrm{~mm}$, $\mathrm{p}=0.001$, respectively) (Fig. 3A). The LV cavity diameter increased by $+21 \%$ in $\mathrm{ED}$ and $+31 \%$ in ES (from $37 \pm 3$ to $45 \pm 3 \mathrm{~mm}, \mathrm{p}=0.001$ and from $30 \pm 4$ to $40 \pm 3 \mathrm{~mm}, \mathrm{p}=0.001$, respectively) (Fig. 3B). Progressive LV dilatation was also documented by MRI, which revealed increase of $+32 \%$ of LV ED volume from $87 \pm 12$ to $115 \pm 21 \mathrm{~mL}$ $(p=0.01)$, and more than doubling of LV ES volume from $29 \pm 4$ to $74 \pm 23 \mathrm{~mL}(\mathrm{p}=0.002)$ (Fig. 3C). Consequently, left ventricular ejection fraction (LVEF) decreased from $66 \pm 3$ to $35 \pm 9 \%$ $(\mathrm{p}=0.0001)$ (Fig. 3D). Switching pacing modes did not affect the measurements of echocardiography, nor of MRI. MRI-derived measurements of LV remodeling are presented in the Supplemental material.

\section{Histological analysis}

The histological analysis of the septal and lateral wall myocardium is presented in the Supplemental material. In brief, lateral wall thickening was confirmed by hypertrophy of the lateral wall cardiomyocytes versus the septal wall cardiomyocytes, while no difference in fibrosis content was observed between both walls.

\section{Impact of acute conduction changes on $\mathrm{LV}$ function in remodeled hearts Global LV function}

Hemodynamic assessment after 8 weeks of rapid pacing revealed $-27 \%$ decrease in $\mathrm{LV}$ global stroke work when switching from AAI to DDD, as determined by the change of area of the pressure-volume loops from $2060 \pm 933$ to $1503 \pm 690$ $\mathrm{mmHg} / \mathrm{mL}(\mathrm{p}=0.034)$ (Suppl. Fig. 2). The $\mathrm{dP} /$ $/ \mathrm{dt}_{\max }$, decreased by $-25 \%$ from $1197 \pm 440$ to $918 \pm 327 \mathrm{mmHg} / \mathrm{s}(\mathrm{p}=0.025)$. CO, MAP and SVR are presented in Supplemental Table 1.

\section{Regional LV function}

Switching from AAI (Fig. 4A) to DDD (Fig. 4B) had a differential effect on the regional circumfer- 


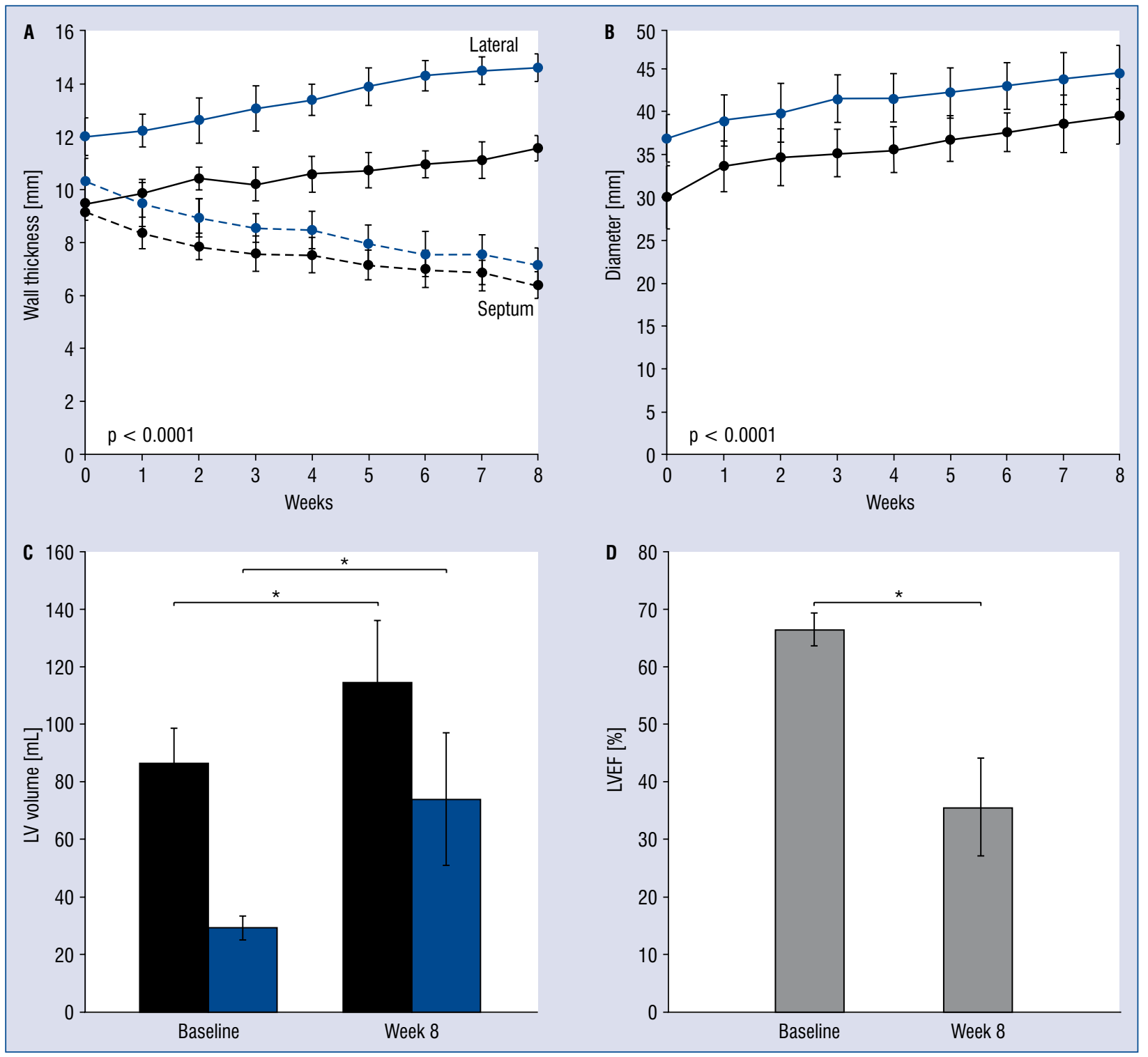

Figure 3. Left ventricular (LV) remodeling during 8 weeks of dyssynchronous rapid pacing in all animals ( $\mathrm{n}=13$ ). Changes in LV septal and lateral wall thickness (A), and LV internal diameter (B), as measured by echocardiography. Changes in LV end-diastolic and end-systolic volume (C), and left ventricular ejection fraction (LVEF) (D) between baseline and week 8 , assessed by cardiac magnetic resonance imaging. Solid lines on the upper panels represent the lateral wall, while dashed lines represent the septal wall measurements. Blue coloured markings are end-systolic, while black coloured markings are end-diastolic measurements. Reported observations were made at $110 \mathrm{bpm}$; ${ }^{*} \mathrm{p}<0.05$.

ential deformation. While septal and lateral wall performed simultaneously in AAI, during DDD, early shortening in the septal region was observed (septal flash), which also pre-stretched the lateactivated lateral wall. Later in systole, the septum performs strongly reduced shortening, followed by a second phase of systolic lengthening, whereas the lateral wall performs pronounced late systolic shortening until aortic valve closure.
Likewise, pressure-strain loops had similar loop areas $(\mathrm{p}=\mathrm{NS})$ in all segments in AAI mode (Fig. 4C, E), indicating homogeneous work distribution. During DDD pacing, regional work increased by $+41 \%$ in lateral wall (from $661 \pm 272$ to $943 \pm 374 \mathrm{mmHg} / \%$, $\mathrm{p}=0.005$ ) and decreased by $-69 \%$ in septum (from $611 \pm 146$ to $190 \pm 139 \mathrm{mmHg} / \%, \mathrm{p}<0.0001)$. Accordingly, septal wall loop area was significantly lower than lateral loop area $(\mathrm{p}<0.0001)$, with septal 


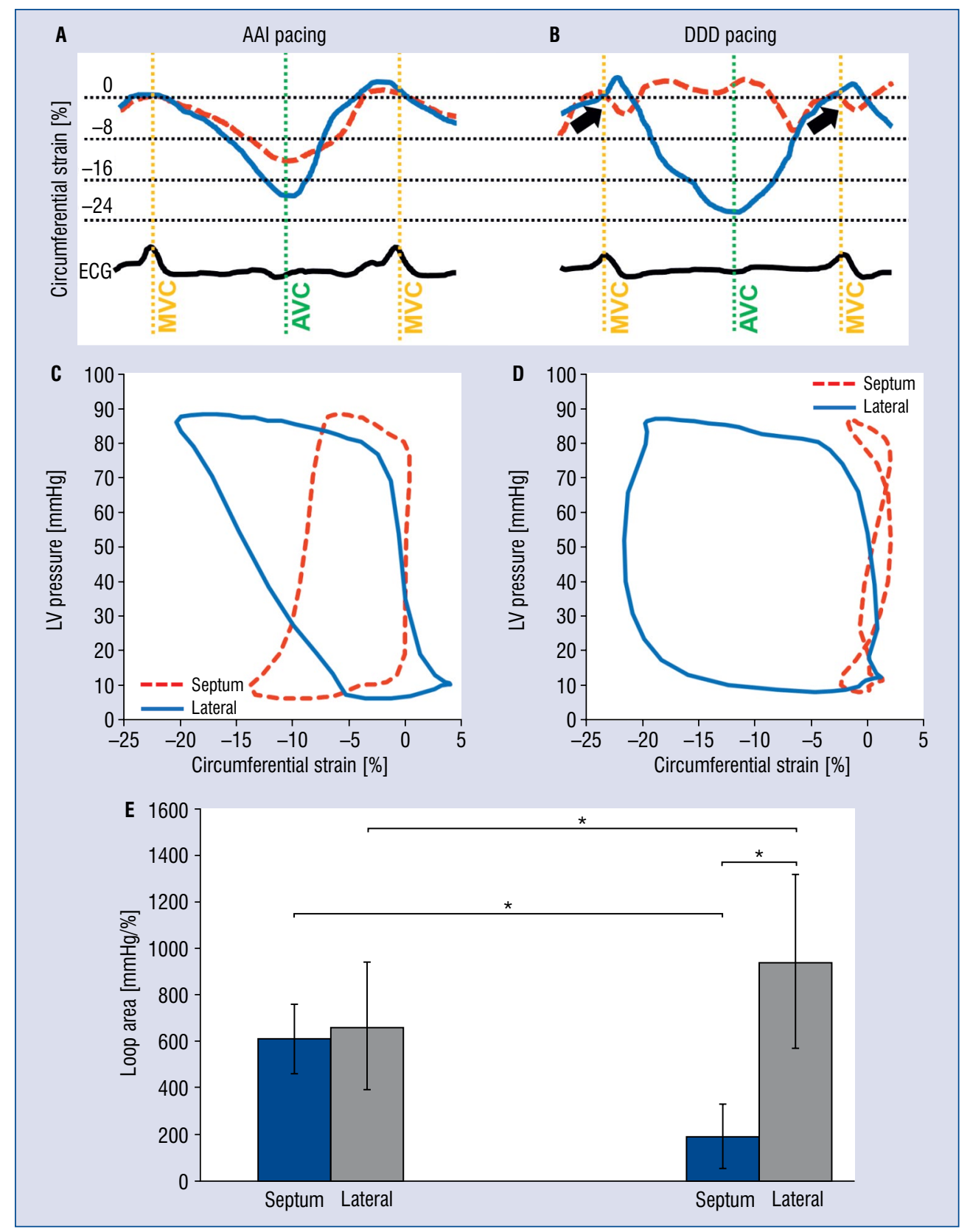

Figure 4. Representative regional circumferential strain curves at mid-ventricular level (A, B), corresponding pressurestrain loops $(\mathbf{C}, \mathbf{D})$, and individual loop areas of all animals $(n=12)(E)$, during AAl pacing (left) and during DDD pacing (right); A, B. During DDD pacing, the septal segment exerts an early septal flash (arrows), while the late-activated lateral wall is pre-stretched; C, D. Note the figure-eight-shaped morphology and small loop of the septal pressurestrain loop during DDD pacing, denoting the low amount of work performed by the septum. During AAl pacing loops retain their normal O-shaped morphology; E. This can also be appreciated in the corresponding measured loop areas. Reported observations were made at $110 \mathrm{bpm}$; ${ }^{*} \mathrm{p}<0.05$; AVC - aortic valve closure; ECG - electrocardiogram; LV - left ventricle; MVC - mitral valve closure.

wall loop frequently showing a figure eight pattern, indicative of work wasting in this region (Fig. 4D, E).

\section{Mitral valve regurgitation}

Before rapid pacing, 7 animals had no mitral regurgitation (MR), 4 had minimal MR, and 2 had mild MR. After 8 weeks of rapid DDD pacing, no animals had no or minimal MR, 7 had mild MR, 3 had moderate MR, and 3 had moderate to severe MR. The average MR grade increased from $0.3 \pm$ \pm 0.26 to $1.69 \pm 0.9(\mathrm{p}<0.0001)$. Switching pacing mode did not affect MR, not at baseline, nor 
after 8 weeks of rapid DDD pacing. Assessment of the LA volume revealed significant increase from $32 \pm 4$ to $81 \pm 35 \mathrm{~mL}(\mathrm{p}=0.001)$. Linear regression analysis revealed that the increase in MR score between baseline and after 8 weeks of rapid DDD pacing and was significantly correlated to the increase in LV ED volume $(\mathrm{r}=0.76, \mathrm{p}=0.002)$ and LA volume $(r=0.83, p=0.001)$.

\section{Reproducibility}

The echocardiographic wall thicknesses and diameter measurements had very good intraobserver variability with an ICC of $0.996,95 \%$ CI 0.993-0.998. Intra- and inter-observer variability of regional strain measurements was good with ICC of $0.951,95 \%$ CI $0.932-0.965$ and $0.95,95 \%$ CI 0.938-0.96, respectively. Pressure-strain loop area measurements had good intra-observer variability with ICC of $0.925,95 \%$ CI 0.903-0.943.

\section{Discussion}

In the present animal model, chronic rapid sequential $\mathrm{RA}$ and $\mathrm{RV}$ pacing was used to induce $\mathrm{LV}$ asymmetrical remodeling was used, and regional inhomogeneities in LV myocardial work which are controllable and reversible by switching pacing mode. Sheep were often regarded as less suited for dyssynchrony research because their conduction system is fast and achievable QRS delays are rather small [2]. Nevertheless, this study demonstrates that sequential RA and RV pacing leads to a doubling of the QRS width and, consequently, to morphologic and functional changes which are closely comparable to other animal models. Although results might differ from human pathology of myopathic hearts suffering from LBBB, it shows comparable functional hallmarks. To our knowledge, this is the first chronic sheep model where all these features are combined and where the functional parameters are shown to be easily controllable.

\section{Left ventricular conduction}

In the present model, sequential RA and RV pacing to induce conduction delay with regionally inhomogeneous myocardial activation was used with the aim of modifying regional myocardial loading. The advantage of this approach over ablation of left bundle branch lies in its reversibility, allowing acute changes in regional wall loading and function by programming the pacemaker. Although the achieved conduction delay appears small compared to other animal models, it is as significant given the much faster conduction in the ovine heart [19]. Near doubling of QRS width during DDD pacing was measured, which is comparable to previously reported values in animal models of ablation or RV pacing in dogs [9] and pigs [20,21]. An overview explaining the major differences between RV pacing and ablation of left bundle branch is presented in Supplemental Table 2.

A small but significant further increase of QRS width with ongoing LV remodeling was also noticed, which can most likely be explained by an effect of remodeling on the conduction system itself [11]. Such 'electrical' remodeling has been suggested as the cause of slow trans-septal conduction in the thinned septum after long-lasting dyssynchrony [13, 14].

\section{Myocardial mechanics}

Compared to AAI, DDD pacing induced LBBB-like myocardial deformation patterns with early septal contraction (septal flash) with subsequent systolic stretching due to lateral wall contraction. Comparable effects have been shown in other animal models [16, 22-24] and patients [24]. Furthermore, DDD pacing decreased LV stroke work and $\mathrm{dP} / \mathrm{dt}_{\max }$ as measured by invasive pressure-volume loops, by $-27 \%$ and $-25 \%$, respectively, which is comparable to canine models of LBBB by ablation $[23,25]$ and findings in human hearts $[24,26]$.

In the present model, performed regional work during AAI pacing was similar in septal and lateral segments (Fig. 4). DDD pacing resulted in significantly lower regional myocardial work in early-activated septal wall segments, whereas late-activated lateral wall segments showed higher work when compared to AAI pacing. The measured effects are in line with previously reported acute changes in animal models before and after ablation [9, 27, 28]. The present chronic model demonstrated that sequential RA and RV pacing can induce very similar effects as in ablation with the advantage that these effects can be reversed by simple pacemaker programming.

\section{Myocardial remodeling by imaging}

In the present model, the chronic asynchronous electrical activation of $\mathrm{LV}$, supported by rapid pacing, induced $-30 \%$ septal thinning and $+22 \%$ lateral wall thickening within 8 weeks. This is advantageous compared to earlier models where remodeling took 3-6 months of $\mathrm{LV}$ pacing at physiological heart rate (and did not resemble the wall thickness distribution in human pathology $[10,11]$ ) or after ablation [9]. Furthermore, it is advanta- 
geous over approaches which apply aortic banding which requires additional surgical skills and leads to extra loading of the heart [29].

The 8 weeks of rapid DDD pacing in this study were also associated with significant increase in MR. Functional MR has been reported in previous animal models of rapid pacing [30] and has been attributed to LV dilatation. This is confirmed by our animal model, where the degree of MR was positively correlated with LV and LA volume while no change was observed when pacing mode was switched between AAI and DDD.

\section{Myocardial remodeling by histology}

Histological analysis of septal and lateral wall samples confirms the hypertrophied appearance of the lateral wall. However, the relative change in the number of transected cardiomyocytes appears to be less pronounced than the change in wall thickness as seen on both echocardiography and MRI. This confirms earlier findings in the canine model [11], and suggests that increased cardiomyocyte length or number could have contributed to the macroscopic increase in lateral wall thickness. In the septum, a possible explanation of the septal thinning could be found in the dilatation of the LV chamber, or a more vertical orientation of myocardial fibre sheets as a result of the long lasting reduced work [14].

No difference in the fraction of collagen and vessels was found between the septal and lateral wall. This implies that no fibrosis or local ischemia was induced during the 8 weeks of rapid DDD pacing. This is in agreement with other publications, where even longer pacing did not induce fibrosis or lead to histological differences with control animals [11].

\section{Limitations of the study}

Although chronic animal models such as the one presented here resemble a clinical situation more correctly than acute animal models, extrapolation of results to patients should be done with caution. While animals present similar phenotypical and functional characteristics as in human pathology, disease history is shorter and underlying pathophysiology might differ from patients.

The scope of this study was not focussed on investigating possible reverse remodeling after cessation of rapid pacing. Nevertheless, other studies have shown that pacing at intrinsic heart rate is able to sustain the remodeling process $[10,11]$, at the expense of much longer development times.
These findings thereby suggest that only cessation of pacing (not rapid pacing) might halt and possibly reverse the remodeling process.

The sample size used in this study was small, which is inherent to fundamental animal research. However, the magnitude of observed changes was comparable to other studies in the field and effects were highly significant.

Mitral regurgitation was qualitatively assessed using MRI. Doppler echocardiography on the other hand, would have been a more quantitative approach. However, sheep have a limited apical acoustic window, making apical images, and therefore correct Doppler tracings difficult and inconsistent.

\section{Conclusions}

This study presents the development of a sheep model that allows for reversible conduction delay and controllable work in an asymmetrically remodelled LV, confirmed by histology. To do so, we applied chronic rapid sequential RA and RV pacing, to avoid irreversible ablation. Re-programing the pacemaker allowed to induce significant immediate changes in regional myocardial function and work. This model provides a valuable alternative to canine or swine models, as it is relatively easy-to-use, slow growing (compared to swine), cheap (compared to minipigs) and leads to less ethical concerns (compared to dogs). It has the potential to become instrumental for investigating the impact of regional function and loading on LV myocardial remodeling.

\section{Acknowledgements}

This work was supported by a KU Leuven research grant (OT/12/084). The Research Foundation - Flanders (FWO) supported K.V. as a post-doctoral fellow, and O.G. and J.U.V. as senior clinical investigators. E.D.P. received a research grant of the European Association of Cardiovascular Imaging.

The authors are indebted to David Célis for his excellent technical assistance during the experiments and care of the animals, We also wish to thank Paul van de Voorde (Medtronic Belgium) for his continuous interest and technical support. Stéphanie Bézy, Marta Cvijic, Serkan Ünlü, and Blazej Michalski have our gratitude for their assistance with preparation of the manuscript.

Conflict of interest: None declared 


\section{References}

1. Houser SR, Margulies KB, Murphy AM, et al. American Heart Association Council on Basic Cardiovascular Sciences, Council on Clinical Cardiology, and Council on Functional Genomics and Translational Biology. Animal models of heart failure: a scientific statement from the American Heart Association. Circ Res. 2012; 111(1): 131-150, doi: 10.1161/RES.0b013e3182582523, indexed in Pubmed: 22595296.

2. Strik M, van Middendorp LB, Vernooy K. Animal models of dyssynchrony. J Cardiovasc Transl Res. 2012; 5(2): 135-145, doi: 10.1007/s12265-011-9336-5, indexed in Pubmed: 22130900.

3. Conn PM. Advantages and Disadvantages of Using Large Animal Models.. Anim. Model. Study Hum. Dis. 2017: 94-95.

4. Cost analysis and rate setting manual for animal research facilities. Lab Anim (NY). 2001; 30(1): 15-16, indexed in Pubmed: 11385721.

5. Fallavollita JA, Riegel BJ, Suzuki G, et al. Mechanism of sudden cardiac death in pigs with viable chronically dysfunctional myocardium and ischemic cardiomyopathy. Am J Physiol Heart Circ Physiol. 2005; 289(6): H2688-H2696, doi: 10.1152/ajpheart.00653.2005, indexed in Pubmed: 16085676.

6. Lionetti V, Guiducci L, Simioniuc A, et al. Mismatch between uniform increase in cardiac glucose uptake and regional contractile dysfunction in pacing-induced heart failure. Am J Physiol Heart Circ Physiol. 2007; 293(5): H2747-H2756, doi: 10.1152/ ajpheart.00592.2007, indexed in Pubmed: 17704291

7. Soliman OII, Geleijnse ML, Theuns DA, et al. Reverse of left ventricular volumetric and structural remodeling in heart failure patients treated with cardiac resynchronization therapy. Am J Cardiol. 2008; 101(5): 651-657, doi: 10.1016/.amjcard.2007.10.027, indexed in Pubmed: 18308015.

8. Thambo JB, Bordachar P, Garrigue S, et al. Detrimental ventricular remodeling in patients with congenital complete heart block and chronic right ventricular apical pacing. Circulation. 2004; 110(25): 3766-3772, doi: 10.1161/01.CIR.0000150336.86033.8D, indexed in Pubmed: 15583083.

9. Vernooy K, Verbeek XA, Peschar M, et al. Left bundle branch block induces ventricular remodelling and functional septal hypoperfusion. Eur Heart J. 2005; 26(1): 91-98, doi: 10.1093/eurhearti/ehi008, indexed in Pubmed: 15615805.

10. Prinzen FW, Cheriex EC, Delhaas T, et al. Asymmetric thickness of the left ventricular wall resulting from asynchronous electric activation: a study in dogs with ventricular pacing and in patients with left bundle branch block. Am Heart J. 1995; 130(5): 1045-1053, indexed in Pubmed: 7484735.

11. van Oosterhout MF, Prinzen FW, Arts T, et al. Asynchronous electrical activation induces asymmetrical hypertrophy of the left ventricular wall. Circulation. 1998; 98(6): 588-595, indexed in Pubmed: 9714117.

12. Leclercq C. Systolic Improvement and Mechanical Resynchronization Does Not Require Electrical Synchrony in the Dilated Failing Heart With Left Bundle-Branch Block. Circulation. 2002; 106(14): 1760-1763, doi: 10.1161/01.cir.0000035037.11968.5c.

13. Strik M, van Deursen CJM, van Middendorp LB, et al. Transseptal conduction as an important determinant for cardiac resynchronization therapy, as revealed by extensive electrical mapping in the dyssynchronous canine heart. Circ Arrhythm Electrophysiol. 2013; 6(4): 682-689, doi: 10.1161/CIRCEP.111.000028, indexed in Pubmed: 23873141.

14. Helm PA, Younes L, Beg MF, et al. Evidence of structural remodeling in the dyssynchronous failing heart. Circ Res. 2006; 98(1): 125-132, doi: 10.1161/01.RES.0000199396.30688.eb, indexed in Pubmed: 16339482.

15. Byrne MJ, Raman JS, Alferness CA, et al. An ovine model of tachycardia-induced degenerative dilated cardiomyopathy and heart failure with prolonged onset. J Card Fail. 2002; 8(2): 108-115, indexed in Pubmed: 12016635.
16. Delhaas T, Arts T, Prinzen FW, et al. Regional fibre stress-fibre strain area as an estimate of regional blood flow and oxygen demand in the canine heart. J Physiol. 1994; 477 ( Pt 3): 481-496, indexed in Pubmed: 7932236.

17. Urheim S, Rabben SI, Skulstad H, et al. Regional myocardial work by strain Doppler echocardiography and LV pressure: a new method for quantifying myocardial function. Am J Physiol Heart Circ Physiol. 2005; 288(5): H2375-H2380, doi: 10.1152/ ajpheart.00946.2004, indexed in Pubmed: 15637114.

18. Forrester JS, Tyberg JV, Wyatt HL, et al. Pressure-length loop: a new method for simultaneous measurement of segmental and total cardiac function. J Appl Physiol. 1974; 37(5): 771-775, doi: 10.1152/jappl.1974.37.5.771, indexed in Pubmed: 4436206.

19. Ono N, Yamaguchi T, Ishikawa H, et al. Morphological varieties of the Purkinje fiber network in mammalian hearts, as revealed by light and electron microscopy. Arch Histol Cytol. 2009; 72(3): 139-149, indexed in Pubmed: 20513977.

20. Rigol M, Solanes N, Fernandez-Armenta J, et al. Development of a swine model of left bundle branch block for experimental studies of cardiac resynchronization therapy. J Cardiovasc Transl Res. 2013; 6(4): 616-622, doi: 10.1007/s12265-013-9464-1, indexed in Pubmed: 23636845.

21. Salameh A, Dhein S, Blanke K, et al. Right or Left Ventricular Pacing in Young Minipigs With Chronic Atrioventricular Block: Long-Term In Vivo Cardiac Performance, Morphology, Electrophysiology, and Cellular Biology. Circulation. 2012; 125(21): 2578-2587, doi: 10.1161/circulationaha.111.079087.

22. Prinzen FW, Augustijn CH, Arts T, et al. Redistribution of myocardial fiber strain and blood flow by asynchronous activation. Am J Physiol. 1990; 259(2 Pt 2): H300-H308, doi: 10.1152/ ajpheart.1990.259.2.H300, indexed in Pubmed: 2386214.

23. Gjesdal O, Remme EW, Opdahl A, et al. Mechanisms of abnormal systolic motion of the interventricular septum during left bundlebranch block. Circ Cardiovasc Imaging. 2011; 4(3): 264-273, doi: 10.1161/CIRCIMAGING.110.961417, indexed in Pubmed: 21393502.

24. Breithardt OA, Stellbrink C, Herbots L, et al. Cardiac resynchronization therapy can reverse abnormal myocardial strain distribution in patients with heart failure and left bundle branch block. J Am Coll Cardiol. 2003; 42(3): 486-494, indexed in Pubmed: 12906978.

25. Vernooy K, Cornelussen RNM, Verbeek XA, et al. Cardiac resynchronization therapy cures dyssynchronopathy in canine left bundle-branch block hearts. Eur Heart J. 2007; 28(17): 2148-2155, doi: 10.1093/eurheartj/ehm207, indexed in Pubmed: 17611254.

26. Auricchio A, Stellbrink C, Block M, et al. Effect of pacing chamber and atrioventricular delay on acute systolic function of paced patients with congestive heart failure. The Pacing Therapies for Congestive Heart Failure Study Group. The Guidant Congestive Heart Failure Research Group. Circulation. 1999; 99(23): 2993-3001, indexed in Pubmed: 10368116.

27. Prinzen FW, Hunter WC, Wyman BT, et al. Mapping of regional myocardial strain and work during ventricular pacing: experimental study using magnetic resonance imaging tagging. J Am Coll Cardiol. 1999; 33(6): 1735-1742, indexed in Pubmed: 10334450.

28. Russell K, Eriksen M, Aaberge L, et al. A novel clinical method for quantification of regional left ventricular pressure-strain loop area: a non-invasive index of myocardial work. Eur Heart J. 2012; 33(6): 724-733, doi: 10.1093/eurheartj/ehs016, indexed in Pubmed: 22315346.

29. Moorjani N, Catarino P, El-Sayed R, et al. A pressure overload model to track the molecular biology of heart failure. Eur J Cardiothorac Surg. 2003; 24(6): 920-925, indexed in Pubmed: 14643809.

30. Timek T, Dagum P, Lai D, et al. Pathogenesis of Mitral Regurgitation in Tachycardia-Induced Cardiomyopathy. Circulation. 2001; 104(suppl 1): I-47-I-53, doi: 10.1161/hc37t1.094913. 